\title{
Propiedades psicométricas del cuestionario modificado de autismo en la infancia (M-CHAT) en dos instituciones educativas para un diagnóstico precoz de autismo.
}

\author{
Psychometric properties of the Modified Checklist for Autism in Toddlers (M-CHAT) in two educational
} institutions for a early diagnosis of autism.

Daniela L. Hidalgo Medina 1,a,b, Elizabeth Dany Araujo Robles 1,2,,d

\section{RESUMEN}

Dada la importancia de realizar una detección temprana y oportuna de los trastornos del espectro autista, la presente investigación se propuso determinar las propiedades psicométricas del M-CHAT en una población peruana de niños de 18 meses a 9 años. Los resultados indican que, a excepción de dos, los ítems cuentan con una alta significancia en cuanto a la validez de contenido. El instrumento cuenta con niveles altos de sensibilidad y especificidad (0,88 y 0,83 , respectivamente). Las correlaciones ítem-test fueron significativas en 14 de los 21 ítems analizados. La validez concurrente se evaluó mediante la correlación del M-CHAT con el ADOS-2 y una prueba de elección visual, siendo ambas correlaciones no significativas. Finalmente, la consistencia interna de la puntuación total y de dos de las tres dimensiones resultó moderada, siendo baja en la tercera de las dimensiones del instrumento.

PALABRAS CLAVE: Trastorno del espectro autista, M-CHAT, propiedades psicométricas, ADOS-2, sistema portátil costo efectivo.

\section{SUMMARY}

Given the importance of early detection of the autism spectrum disorders, this research had as an objective to determine the validity and reliability of the Modified Checklist for Autism in Toddlers (M-CHAT) in a Peruvian 18 months to 09 years old children sample. Results showed that, except for two items, the most of them have highly significant content validity. This instrument exhibits high levels of sensibility and specificity (.88 and .83 , respectively). Item-test correlations were significant in 14 out of the 21 items submitted to analysis. Concurrent validity was assessed through correlations of M-CHAT with ADOS-2 and a visual election task, both of them were not significant. Finally, internal consistency of total scores and that of two of the three dimensions was moderated, but low in the case of the third dimension.

KEYWORDS: Autism spectrum disorder, M-CHAT, psychometric properties, ADOS-2, cost effective portable system.

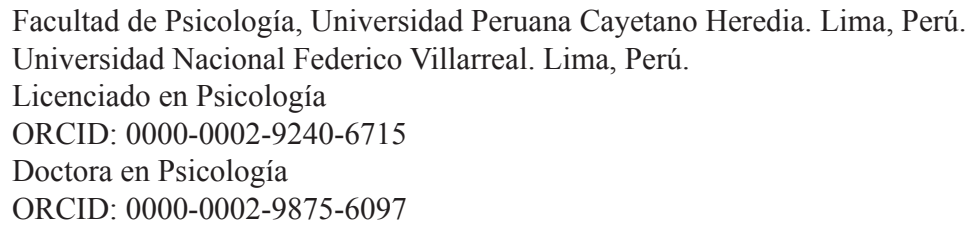




\section{INTRODUCCIÓN}

Los Trastornos de Espectro Autista (TEA) son definidos como una discapacidad del desarrollo, con criterios diagnósticos que incluyen déficits en la comunicación y la interacción social además de restringidos patrones repetitivos de comportamientos, intereses $\mathrm{y}$ actividades (American Psychiatric Association, 2013). Los TEA aparecen en un periodo temprano del desarrollo; sin embargo, los déficits sociales y de comportamiento que los caracterizan no pueden ser reconocidos como síntomas de TEA hasta que el niño se encuentre en un ambiente educativo y social. Las limitaciones funcionales varían entre las personas con TEA y pueden desarrollarse con el tiempo (National Center on Birth Defects and Developmental Disabilities, 2010), estas diferencias dificultan el diagnóstico específico. Sin embargo, en las últimas décadas la práctica clínica, la investigación y los desarrollos teóricos han brindado nuevas herramientas para la evaluación en el campo del autismo, lamentablemente en el Perú no se han desarrollado herramientas diagnósticas que cumplan con los estándares psicométricos requeridos para las evaluaciones.

Los datos epidemiológicos muestran una prevalencia de casos de TEA de 6.5 cada 1000 nacimientos; incluso existen estadísticas que señalan que 1 de cada 68 nacimientos se diagnostican con TEA a nivel mundial (Centers for Disease Control, s. f.), lo que apunta a un nuevo desafío para todos los profesionales de la salud. En la realidad peruana, según el Consejo Nacional de la Igualdad de las Discapacidades (2014), 4 de cada 1000 niños son afectados por autismo y alrededor del $75 \%$ de este grupo presenta algún otro trastorno del desarrollo (retraso mental o motriz, síndrome de Down, entre otros). En el Perú, las personas con autismo son consideradas dentro del grupo de discapacitados físicos, sociales, cognitivos y mentales, constituyendo el $31.4 \%$ de esta población. Frente a esta situación, el 8 de Enero del 2014 se aprobó en el Perú la Ley de Protección de las Personas con TEA, la cual busca alternativas para la detección y el diagnóstico precoz, la intervención temprana, la protección de la salud, la educación integral, la capacitación personal y la inserción laboral y social de las personas con TEA; sin embargo, existe un gran camino que recorrer en relación al trabajo y diagnóstico de estos niños. (Ministerio de la Mujer y Poblaciones Vulnerables, 2014)
Los TEA contienen síntomas muy severos que se identifican mediante un examen neurológico y psicológico, siendo la edad de diagnóstico entre los 5 y 6 años, en nuestro país. Además de ello, las familias de niños con autismo presentan varias dificultades en relación al diagnóstico debido a que son ellos quienes identifican las primeras sospechas en el retraso del desarrollo de su hijo; asimismo, después del diagnóstico tardío estas familias se enfrentan a nuevas dificultades como la falta de información, expresar lo que sucede y ser escuchadas, lo que genera estrés y ansiedad frente al futuro. Según Suárez (1994), los padres mantienen características ansiosas frente a la presencia de un hijo con esta condición.

El impacto del autismo no sólo en el sujeto, sino en la familia y el Estado apunta a una gran necesidad de identificar herramientas para el diagnóstico temprano (Sampedro, 2012). Los TEA y otros trastornos generalizados del desarrollo son difíciles de detectar en niños pequeños ya que los síntomas presentes son muy variables en cada caso, además de que los déficits sociales y retrasos de lenguajes son identificados hasta que el niño es presentado en una situación de interacción, por ejemplo en el caso preescolar. La situación se vuelve más difícil si se carece de un instrumento estandarizado y de fácil administración para niños pequeños dificultando la identificación de casos antes de los tres años de edad. Las investigaciones de los últimos años han perfeccionado la descripción clínica de este trastorno y sus límites clasificatorios aportando nuevos métodos de identificación y de diagnóstico; sin embargo, se carece de biomarcadores específicos, pruebas de laboratorio o instrumentos clínicos que identifiquen objetivamente este trastorno, debido a los rasgos homogéneos y heterogéneos que presentan estos cuadros. Según Díez-Cuervo (2005) el diagnóstico especializado es un procedimiento costoso y complejo, dependiente de un juicio clínico informado.

Es por estas razones que el estado peruano ha creído conveniente invertir en la creación de herramientas costo-efectivas que permitan la identificación de este tipo de trastornos del desarrollo. De este modo, Innovate Perú, un fondo que financia proyectos nacionales, se encuentra apostando por desarrollar una herramienta portátil para la identificación de preferencia visual en niños pequeños, con la cual poder reconocer rangos de preocupación en relación a trastornos del desarrollo; este plan de trabajo se viene realizando desde el año 2015. A partir de estos estudios, se busca estructurar, probar y validar 
una herramienta diagnóstica comparándola con instrumentos psicológicos y con un gold standard para la identificación del autismo en la infancia. Si bien la identificación de los TEA se basa principalmente en las impresiones clínicas basadas en la observación, se han desarrollado varios instrumentos destinados al diagnóstico precoz que son irrenunciables, ya que facilitan la identificación fiable de las características clínicas, cognitivas y conductuales del autismo pero que no se encuentran validadas en el contexto peruano. La presente investigación, que forma parte del proyecto antes mencionado, se planeó para aportar una herramienta psicológica cuyo objetivo es la evaluación de un trastorno que no ha sido investigado aún en el país partiendo de una necesidad urgente por cubrir. Se trata del Cuestionario Modificado de Autismo en la Infancia (M-CHAT), el cual cuenta con las características de practicidad y utilidad como herramienta para el cribado de trastornos del espectro autista.

El proceso requerido para el diagnóstico de los TEA debe cumplir al menos dos niveles de actuaciones; en un primer nivel, conocido como cribado o screening, se trataría de buscar dentro de la población sana infantil una serie de signos de alta probabilidad; esos sujetos que muestran un rango de preocupación pasan a un segundo nivel en donde se llevarían a cabo una serie de pruebas diagnósticas que nos permitan descartar otras patologías, registrar el caso y finalmente tener un diagnóstico clínico (Posada-De la Paz, 2005). En el primer nivel se tiene como propósito detectar manifestaciones sugerentes de la presencia de un TEA, estas manifestaciones se encuentran en alguno de los tres grandes campos de preocupación temprana: comunicación, la interacción social y las conductas. Entre las manifestaciones referidas por los padres, destacan las siguientes: no responder al nombre, no mostrar interés por otros, tener rabietas frecuentes y desproporcionadas, andar de puntillas y tener una adhesión excesiva a algún objeto (Filipek, 1999).

En el DSM-5se eliminó la categoría de Trastornos Generalizados del Desarrollo (American Psychiatric Association, 2013), en cambio, se describe una categoría denominada trastornos del neurodesarrollo, en la cual se encuentran los TEA como único diagnóstico, desapareciendo el diagnóstico diferencial del trastorno autista con el síndrome Asperger, síndrome de Rett, trastorno desintegrativo infantil y autismo no especificado. En esta nueva revisión se definen dos criterios diagnósticos: (a) déficits persistentes en la comunicación y en la interacción social en diversos contextos, no atribuibles a un retraso general del desarrollo y (b) patrones de comportamiento, intereses o actividades restringidas y repetitivas. Además de ello, se sugiere que los síntomas deben estar presentes en la primera infancia y que los síntomas limitan y discapacitan para el funcionamiento cotidiano. En suma, los criterios psiquiátricos existentes para diagnosticar el trastorno autista señalan que este trastorno se caracteriza por un marcado déficit en el área social, para comunicarse con las demás personas o para entenderlas, además de una carencia de empatía y de atención conjunta con las demás personas, aparente rigidez cognitiva que se manifiesta en una intolerancia a cambios en el ambiente, presencia de rituales bizarros o sin sentido aparente y alteraciones a nivel sensorial, estas se caracterizan por una sensibilidad o

Tabla 1. Características de los Trastornos del Espectro Autista

\section{TRASTORNOS DEL ESPECTRO AUTISTA}

\begin{tabular}{ll}
\hline Dimensión & Trastorno \\
& 1. Trastorno de la relación social \\
A. Relaciones Sociales & 2. Trastorno de la referencia conjunta (acción, atención y \\
preocupación conjunta) & 3. Trastorno intersubjetivo y mentalista \\
& 4. Trastorno de las funciones comunicativas. \\
B. Áreas de lenguaje y comunicación & 5. Trastorno del lenguaje expresivo \\
& 6. Trastorno del lenguaje receptivo \\
C. Área de flexibilidad mental y comportamental & 7. Trastorno de la anticipación \\
& 9. Trastorno de la flexibilidad \\
& 10. Trastorno de la ficción \\
D. Área de ficción e imaginación & 11. Trastorno de la imitación \\
& 12. Trastorno de la suspensión.
\end{tabular}

Nota: Tomada de (Riviére, 1997) 
insensibilidad extrema a estímulos sensoriales tales como calor, frío, dolor, entre otros, o por ser inusuales (Villalba, 2013).

Actualmente existen herramientas de cribado específicas de autismo, basadas en la presencia de los síntomas sugestivos de Trastorno General del Desarrollo (TGD) que aparecen a edades muy tempranas como resultado de la afectación de las tres áreas fundamentales implicadas en este trastorno: interacción social, comunicación y patrones repetitivos de comportamiento, intereses y actividades. Aunque las herramientas para la detección temprana de los trastornos del desarrollo han mejorado, se han de tener en cuenta numerosos factores en el momento de seleccionar la más apropiada, teniendo en cuenta factores como disponibilidad, facilidad de manejo, seguridad en características psicométricas del instrumento a utilizar (sensibilidad y especificidad), tiempo empleado en su administración, costeefectividad y adecuación al entorno y al personal que la administre, haciendo que sea posible su manejo por profesionales no especializados. (Canal et al., 2007).

Riviére (1997) propuso cuatro categorías o dimensiones compuestas con cuatro trastornos cada una, las cuales conforman el espectro autista. En la Tabla 1 se describen las dimensiones y trastornos descritos por Riviére, que posteriormente sirvieron para crear el Inventario de Espectro Autista (IDEA).

El M-CHAT (Robins, Fein, Barton \& Green, 2001) es el método usado internacionalmente para detectar TEA en niños con edades comprendidas entre 16 y 30 meses. La razón para seleccionar este instrumento para su validación en este proyecto es que consideramos que es la herramienta con menores necesidades de adaptación al entorno de la atención primaria de salud (cuenta con una traducción en español). Otra característica relevante es que no precisa de la intervención directa del médico especialista, ya que el cuestionario es auto-administrado, sólo requiere del especialista al entregar el cuestionario a la familia y enviarlo a los servicios de interpretación una vez culminado. Se considera que un niño tiene signos de alarma según el cuestionario si falla en dos o más ítems críticos $(2,7,9,13,14,15)$ o si falla en tres ítems cualesquiera. No todos los casos en los que se fallen suficientes ítems tienen por qué cumplir los criterios diagnósticos.

Existen adaptaciones del M-CHAT en España; en donde además se buscó analizar la capacidad discriminativa de este instrumentos entre casos de
TEA y no TEA y analizar la capacidad predictiva de cada uno de los ítems del cuestionario para detectar casos de riesgo de TEA en la población española (Canal et al., 2007). Para determinar la capacidad discriminativa, se utilizó una población de 48 niños, con edad cronológica comprendida entre 18 y 43 meses y una edad mental entre los 14 y 24 meses. El grupo con resultado M-CHAT positivo estaban conformados por 21 varones y 3 mujeres, mientras que en el grupo de niños con resultado de M-CHAT negativo se encontraban 17 niños y 7 niñas. Al analizar la capacidad predictiva se utilizó una muestra de 2.281 niños con una edad cronológica comprendida entre 18 y 43 meses. En dicha investigación se realizó la traducción del instrumento al idioma castellano y se analizaron las dificultades de comprensión de los ítems a través de llamadas telefónicas realizadas a personas que contestaron al cuestionario administrado en las consultas de pediatría. Se envió la lista de adaptaciones a la autora del cuestionario que admitió la incorporación de las modificaciones realizadas. Finalmente, no fue eliminado ningún ítem del cuestionario original, sólo se hicieron adaptaciones referidas al contexto con el fin de facilitar la comprensión lo máximo posible.

En el desarrollo de dicho estudio, el 50\% de los niños participantes eran niños con sospecha de un trastorno grave del desarrollo, procedentes de consultas o centros especializados y el otro $50 \%$ eran niños con desarrollo normal procedentes de las consultas de pediatría de atención primaria. El resultado de comparar la clasificación de los niños de acuerdo al M-CHAT/ES con la clasificación de los niños según los criterios DSM-IV TR deja claro que se cuenta con la capacidad para identificar a todos los niños con riesgo de TEA, aunque también puede clasificar dentro del grupo de niños de riesgo a otros niños con trastornos del desarrollo del lenguaje o cognitivo. Por consiguiente, los resultados se consideraron muy prometedores en cuanto a las posibilidades del instrumento para la detección precoz, aunque no sólo del autismo, sino de trastornos tempranos de la comunicación y la socialización.

Manzone (2013) realizó la adaptación y validación del M-CHAT para la población urbana argentina. El estudio comprendió una muestra de niños de población general $(n=420)$ y a una muestra de niños de población clínica, con TEA $(\mathrm{n}=140)$. La edad cronológica comprendida se hallaba entre los 18 y 24 meses. Aunque el M-CHAT ya había sido traducido al español se procedió a una nueva traducción adaptada a la cultura argentina. La consistencia interna del 
instrumento fue evaluada a través del cálculo de coeficiente Alfa de Cronbach. La comparación de los ítems entre ambas muestras utilizando una tabla de contingencia con la prueba de Chi cuadrado (X2), mostró que había diferencias $(\mathrm{p}<0,001)$ en todos los ítems entre la población general y la población clínica, excepto para el ítem 16. En el análisis de la regresión logística el modelo que presentó el mejor porcentaje global de clasificación de casos positivos y menor de falsos positivos, alcanzó a un $97,5 \%$ correcto de los casos. De esta clasificación se obtuvo una especificidad del 98,8\% y una sensibilidad de 93,6. Los resultados mostraron una buena capacidad predictiva en los ítems 1, 2, 4, 5, 7, 11, 13, 14, 20, 22 y 23. En el Perú no se han encontrado investigaciones sobre la adaptación o las propiedades psicométricas de este instrumento.

\section{MATERIAL Y METODOS}

Los participantes pertenecían a la base de datos del proyecto "Sistema Portátil Costo Efectivo para el diagnóstico precoz de autismo". Para el estudio de validez de contenido, realizada por el criterio de jueces, se aplicó el cuestionario a 10 especialistas en el tema. Las demás partes del estudio psicométrico comprendieron la participación de niños, de la siguiente manera.

A) Estudio de validez clínica. La muestra estuvo constituida por 31 niños, de los cuales 8 fueron denominados casos (niños con un diagnóstico de TEA) con una edad cronológica entre 4 y 9 años de edad; además de 23 niños denominados controles (niños con desarrollo neurotípico) entre las edades de 2 y 6 años de edad.

B) Estudio de validez de constructo. La muestra fue de 78 niños entre los 18 meses y 6 años de edad, todos ellos pertenecientes a dos instituciones educativas de ciclo1 y ciclo 2 . El ciclo 1 se dirige a niños de 90 días a menores de 3 años y tiene como finalidad brindar estimulación requerida y con ello la posibilidad de desarrollarse en forma equilibrada y oportuna, respetando su nivel de madurez. El ciclo 2 está dirigido a niños de 3 a menos de 6 años, y busca brindar actividades técnico pedagógicas y servicios complementarios y compensatorios de salud y alimentación orientados a favorecer el desarrollo bio-psicomotor, intelectual y socioemocional del niño.

C) Estudio de validez concurrente. Se trabajó con una muestra de 31 infantes para el estudio de correlación con el ADOS-2 y con 50 niños para el estudio de la correlación bivariada con el sistema portátil costo efectivo.

D) Finalmente, para la confiabilidad de estabilidad se trabajó con una muestra de 75 niños.

\section{Instrumentos y procedimiento de aplicación}

Cuestionario Modificado de Autismo en la infancia (M-CHAT/ES). Es un instrumento creado con la intención de mejorar la sensibilidad del CHAT, consiste en 23 preguntas de respuestas dicotómicas si-no, de las cuales 9 corresponden al CHAT original y tiene un agregado de 14 preguntas referidas a los síntomas medulares presentes entre los niños con autismo muy pequeños. Se diseñó en los Estados Unidos por Robins et al., (2001) y la parte de observación directa del CHAT original fue omitida. Fue validado, en el mismo país de origen, con una muestra de 2500 niños de 2 años y presenta una sensibilidad de 0,87, una especificidad de 0,99 , un valor predictivo positivo de $80 \%$ y un valor predictivo negativo del $99 \%$ (Robins et al., 2001).

La traducción al castellano de esta prueba fue realizada en España (Canal et al., 2007). El M-CHAT en español incluye los ítems mencionados agregando algunos otros; por ejemplo, anormalidades sensoriales (hipersensibilidad a los ruidos) anormalidades motoras (movimientos raros con los dedos, dificultades para escalar), intercambio social (contacto visual, sonrisa en respuesta a la sonrisa de los padres), atención conjunta primaria/ teoría de la mente (traer objetos para mostrar a los padres, señalar para mostrar interés, seguir al adulto), lenguaje y comunicación (señala para solicitar, entendiendo "no", indicando propios deseos). Además de ello, el objetivo de esta versión fue mejorar la sensibilidad en comparación con el CHAT original por varias razones: (a) la edad de detección es de 24 meses en lugar de 18 con el fin de identificar a los niños que manifiestan regresión entre los 18 y los 24 meses; (b) el M-CHAT tiene un umbral más bajo para seguimiento y (c) el uso de una entrevista telefónica estructurada como una etapa de selección intermedia mantiene la especificidad relativamente alta sin comprometer la sensibilidad (Robins et al., 2001).

En el primer estudio realizado por Robins et al. (2001) se determinó la confiabilidad mediante alpha de Cronbach para los 22 ítems así como para los 6 ítems encontrados como mejores discriminantes para los niños diagnosticados con TEA. La consistencia interna fue adecuada para el total de los ítems $(\alpha=$ $.85)$ y para los ítems críticos $(\alpha=.83)$. Por otro 
lado, el análisis de los ítems utilizando chi cuadrado indicó que todos los ítems fueron significativamente diferentes entre los niños diagnosticados como autistas en comparación con todos los demás participantes. El análisis de función discriminante se realizó utilizando los 22 ítems del M-CHAT originales (sin incluir el ítem 23 de referencia social) con el fin de determinar la capacidad de clasificar a los niños, ya sea como TEA o no TEA. Además de ello se mostraron los coeficientes de la función discriminante canónicos estandarizados para cada ítem, siendo los ítems 7,14,2,9,15 y 13 los que obtuvieron el peso más alto.

Escala de Observación para Diagnóstico de Autismo (ADOS-2). Esta escala está estructurada en cinco módulos (T, 1, 2, 3 y 4) y su objetivo es la evaluación estandarizada y semiestructurada de la comunicación, la interacción social y el juego o el uso imaginativo de materiales para personas con sospecha de tener un TEA. Cada módulo está destinado a personas con una edad cronológica y un nivel de lenguaje determinado, como se indica a continuación: Módulo T: Para niños pequeños que tienen entre 12 y 30 meses de edad y que no usan de manera consistente un lenguaje de frases.

Módulo 1: Para niños que tienen 31 meses o más y que no usan de manera consistente un lenguaje de frases.

Módulo 2: Para niños de cualquier edad que usan un lenguaje de frases, pero que no tienen fluidez verbal.

Módulo 3: Para niños con lenguaje fluido y adolescentes jóvenes (menores de 16 años).

Módulo 4: Para adultos y adolescentes de 16 años en adelante con lenguaje fluido.

Tras corregir el protocolo y obtener las puntuaciones del algoritmo, se realiza el diagnostico a partir de los puntos de corte establecidos.

En la presente investigación se utilizó el módulo $\mathrm{T}$, aplicable para niños entre 12 a 30 meses de edad y cuya finalidad es otorgar un rango de preocupación específico. De los 41 ítems del módulo $\mathrm{T}, 39$ tuvieron un acuerdo exacto igual o superior a $80 \%$ considerado como bueno en función del porcentaje de acuerdo interjueces. En relación a la fiabilidad de las puntuaciones de los dominios y la clasificación, se calcularon correlaciones intraclase para las puntuaciones de algoritmo total global y las puntuaciones totales de los dominios afectación social y comportamientos restrictivo y repetitivo, así como la suma total de todos los ítems de la sección codificación de protocolo.

En relación a la fiabilidad test-retest, se abordó utilizando los datos de todos los niños a los que se les aplicó el módulo T; se analizó utilizando la puntuación total global del algoritmo y las puntuaciones totales de los dominios afectación social y comportamientos restringidos y repetitivos. Las correlaciones fueron las siguientes: 0,88 (total global del algoritmo), 0,87 (total dominio Afectación social) y 0,64 (total del dominio comportamientos restringidos y repetitivos) (Lord et al., 2015).

Sistema portátil costo efectivo. Esta herramienta está basada en la tecnología de seguimiento de los ojos ("eye tracking") que busca identificar los primeros cambios en la preferencia de la mirada a seguir escenas sociales. Se utilizó un video con la pantalla dividida en dos escenas, donde una escena estaba compuesta por niños bailando y la segunda escena estaba compuesta por grabaciones de protectores de pantalla formada por curvas abstractas en movimiento. Los niños con TEA tienden a centrar su atención en los objetos inertes, más que en las personas; comparado con un niño de desarrollo neurotípico que centra más su atención en las personas pues es así como desarrollan herramientas de comunicación con sus pares. Los niños identificados con preferencias hacia la repetición geométrica son candidatos para más evaluaciones del desarrollo y un tratamiento temprano. El descubrimiento de una preferencia temprana para la repetición geométrica se mueve más allá de los déficits sociales más comúnmente estudiados, abriendo una línea de investigación sobre el desarrollo temprano de estas características en el autismo.

El software desarrollado para el diagnóstico temprano de TEA en niños pequeños, tiene como objetivo principal rastrear el movimiento de los ojos y la fijación de la mirada de los niños, utilizando como método de rastreo el procesamiento de imágenes de un video. El vector entre el centro de la pupila y los reflejos corneales se utiliza para calcular el punto de consideración en la superficie o en la dirección de la mirada, este punto influye de manera directa en el rendimiento como en la precisión del sistema. Además, el sistema debe ser portátil, accesible, de uso fácil y bajo costo. A continuación se describirán a detalle las partes del algoritmo que se tomaron en cuenta:

1. Datos personales. El software recabó datos personales del paciente como edad, peso, talla, sexo, entre otros, para generar una historia médica 
electrónica.

2. Grabación del movimiento ocular. Esta parte del proceso es la más importante, debido a que el vídeo del movimiento visual del niño es la entrada para el algoritmo "eye-tracking”. Se realizó la grabación a través de la webcam del dispositivo electrónico, luego se dividió el video en frames y se analizó cada frame para deducir la preferencia visual del niño.

3. Reproducción del vídeo. Se desarrolló un vídeo de un minuto de duración, que consta con una introducción de 10 segundos y con una pantalla dividida con dos escenas, una social y la otra de objetos en los restantes 50 segundos.

4. Algoritmo. Este proceso dentro del diagrama de bloques fue el encargado de tomar las entradas, que en este caso fueron los frames del video de los ojos del niño. Para llegar al resultado, se realizaron tres etapas: la etapa de preprocesamiento de las imágenes, la etapa del procesamiento propiamente dicho y la etapa de extracción de las características, que es la etapa que recopiló la información de la fotografía de interés.

5. Servidor Web. En esta parte del proceso es donde se recopiló toda la información de las pruebas realizadas y se realizó la etapa de procesamiento del algoritmo, ya que como se trabajó en el procesamiento de imágenes se necesitó un buen procesador y una buena memoria para esta tarea.

En las pruebas piloto y de validación del software se encontró que el algoritmo filtra correctamente la preferencia visual del niño con un nivel de fiabilidad $(\mathrm{p}<.05)$ comparado con un gold standard.

\section{Procedimiento general}

Para los datos del primer cribado, se utilizó M-CHAT y el sistema portátil costo efectivo. Se procedió a realizar el análisis de los resultados además de identificar a los niños que "fallaron" en la aplicación haciendo un seguimiento telefónico para confirmar respuestas con padres y/o cuidadores. Finalmente, y en caso fallaran, se aplicó la prueba diagnóstica ADOS-2 para tener una estimación del riesgo (se contó con una certificación en investigación para la aplicación de esta última prueba psicológica).

Las puntuaciones de los instrumentos se calificaron según la fundamentación de cada prueba, de la siguiente manera:

A) M-CHAT: El nivel de riesgo de tener TEA en función a fallar dos de los seis ítems críticos o tres de los demás ítems. Las respuestas son categorizadas como 0 cuando no cumple con los criterios, 1 cuando falla los ítems y 2 cuando ha fallado a un número mayor de 6 ítems.

B) ADOS-2: Rangos de preocupación en función a todos los niños pequeños/niños mayores con pocas palabras o ninguna (12 a 20 meses) y Niños mayores con algunas palabras (21 a 30 meses) en niveles de Moderada-Severa (2), Leve-Moderada (1) y Poca-Ninguna (0).

C) Sistema portátil costo efectivo: Rango de preferencia en función a escenas sociales y escenas abstractas.

Se utilizó el procedimiento de jueces expertos para determinar si los ítems de las dimensiones del M-CHAT son representativos de estos o universo del contenido. Para ello, se envió la escala a 10 psicólogos con experiencia de atención a pacientes con diagnóstico de autismo; los expertos recibieron información acerca del propósito de la prueba.

\section{RESULTADOS}

El estudio de jueces demostró una concordancia del $100 \%$ entre jueces para un total de cinco ítems, una concordancia del $90 \%$ entre jueces para un total de ocho ítems, una concordancia de $80 \%$ entre jueces para un total de ocho ítems y finalmente una concordancia de $70 \%$ entre jueces para un total de dos ítems.

La dimensión A, denominada alteraciones en el desarrollo de la interacción social, la respuesta emocional y el juego" incluye indicadores que han obtenido un coeficiente de Aiken que oscila entre

Tabla 2. Coeficientes V de Aiken en la dimensión A.

\begin{tabular}{cccc}
\hline Ítems & Coeficiente $\mathbf{~}$ & $\boldsymbol{p}$ & Nivel \\
\hline ÍTEM 1 & 0,8 & 0,049 & Alto \\
ÍTEM 2 & 0,8 & 0,049 & Alto \\
ÍTEM 3 & 0,9 & 0,001 & Alto \\
ÍTEM 4 & 0,9 & 0,001 & Alto \\
ÍTEM 5 & 0,9 & 0,001 & Alto \\
ÍTEM 8 & 0,8 & 0,049 & Alto \\
ÍTEM 10 & 1 & 0,001 & Perfecto \\
ÍTEM 12 & 0,8 & 0,049 & Alto \\
ÍTEM 13 & 0,9 & 0,001 & Alto \\
ÍTEM 14 & 1 & 0,001 & Perfecto \\
\hline Nota: Coeficiente $V=$ de Aiken & &
\end{tabular}


0.80 hasta 1.0; además de señalar que todos los ítems presentan un nivel de significación menor a .05 por lo que se pueden considerar como estadísticamente significativos en relación a esta dimensión (Tabla 2).

$\mathrm{La}$ dimensión $\mathrm{B}$, denominada, retraso o anormalidades en el desarrollo del lenguaje y la comunicación, incluye indicadores que han obtenido un coeficiente de Aiken que oscila entre 0,70 hasta 1,0; además la mayoría de los ítems presentan un nivel de significación menor a 0,05 , por lo que se pueden considerar como estadísticamente significativos en relación a esta dimensión, sólo los ítems 17 y 21 no han alcanzado los criterios requeridos para ser considerados como estadísticamente significativos (tabla 3).

Tabla 3. Coeficientes V de Aiken en la dimensión B.

\begin{tabular}{cccc}
\hline Ítems & Correlación $\mathbf{V}$ & $\mathbf{P}$ & Nivel \\
\hline ÍTEM 6 & 0,9 & 0,001 & Alto \\
ÍTEM 7 & 0,9 & 0,001 & Alto \\
ÍTEM 9 & 0,9 & 0,001 & Alto \\
ÍTEM 15 & 0,8 & 0,049 & Alto \\
ÍTEM 17 & 0,7 & & Alto \\
ÍTEM 19 & 0,8 & 0,049 & Alto \\
ÍTEM 20 & 1 & 0,001 & Perfecto \\
ÍTEM 21 & 0,7 & & Alto \\
ÍTEM 23 & 0,9 & 0,001 & Alto \\
\hline Nota: Coeficientes V = de Aiken & &
\end{tabular}

En la dimensión C, llamada intereses restringidos, movimientos repetitivos y alteraciones motrices, se han encontrado indicadores que presentan coeficientes de Aiken que oscilan entre 0.80 hasta 1.0; todos los ítems presentan un nivel de significación menor a .05 por lo que se pueden considerar como estadísticamente significativos (tabla 4).

Tabla 4. Correlación V de Aiken en la dimensión C.

\begin{tabular}{cccc}
\hline Ítems & Correlación $\mathbf{V}$ & $\boldsymbol{p}$ & Nivel \\
\hline ÍTEM 11 & 1 & 0,001 & Perfecto \\
ÍTEM 15 & 0,8 & 0,049 & Alto \\
ÍTEM 17 & 1 & 0,001 & Perfecto \\
ÍTEM 22 & 0,8 & 0,049 & Alto \\
\hline
\end{tabular}

Nota: Coeficientes $V=$ de Aiken

En cuanto a la validez clínica, la Tabla 5 muestra la distribución de frecuencias de niños con TEA y sin TEA identificados como casos positivos o negativos.
A partir de esos resultados, se puede identificar la proporción del total de niños con TEA que el M-CHAT es capaz de detectar, denominado índice de sensibilidad, que en este caso asciende a .88 . Además, se puede identificar la utilidad de la prueba para identificar a los niños NO TEA señalado por el índice de especificidad, el cual alcanzó un valor de 0,83 . Por otro lado, podemos agregar a este análisis el valor predictivo positivo (VPP) de 0,64 haciendo referencia a la probabilidad de padecer la enfermedad si se obtiene un resultado positivo en la prueba y un valor predictivo negativo (VPN) de .95 indicando la probabilidad de que un sujeto con un resultado negativo en la prueba esté realmente sano.

Tabla 5. Distribución de casos positivos y negativos en niños con y sin TEA

\begin{tabular}{ccccc}
\hline & & Niño & Niño & \\
& & NO & Total \\
\cline { 2 - 5 } MCHAT & Positivo & VP & FP & \\
& & 7 & 4 & 11 \\
& Negativo & FN & VN & 20 \\
& & 1 & 19 & \\
& Total & 8 & 23 & 31 \\
\hline
\end{tabular}

Nota: $V P=$ Verdadero Positivos, $V N=$ Verdaderos Negativos, $F N=$ Falsos Negativos y FP=Falsos Positivos

Para determinar la validez de constructo, se realizó el análisis de ítems mediante la correlación ítem test. La tabla 6 muestra los resultados. Algunos de los ítems presentaron correlaciones de un nivel nulo (ítem 1 , ítem 2, ítem 4, ítem 13, ítem 15 , ítem 20 e ítem 21), otros presentaron correlaciones débiles (ítem 8 , ítem 11, ítem 12, ítem 15, ítem 17, ítem 18 e, ítem 19) y algunos otros, de un nivel moderado (ítem 3 , ítem 5, ítem 6, ítem 7, ítem 9, ítem 10, ítem 16 e ítem 23). En el caso de dos ítems (ítems 14 e ítem 22), las correlaciones no pudieron ser calculadas puesto que sus resultados fueron constantes. Debido a esto último no fue posible realizar un análisis factorial de las puntuaciones.

Para determinar la validez concurrente se calcularon las correlaciones de las puntuaciones del M-CHAT con las de ADOS-2 y del sistema portátil costo efectivo. Con el ADOS-2, se encontró una correlación positiva de nivel moderado de $.306(p=0,094)$. La correlación con la puntuación del sistema portátil costo efectivo fue de $.024(p=0,871)$.

Por último, se calculó la confiabilidad de las puntuaciones del M-CHAT mediante consistencia 
Tabla 6. Correlaciones item-test del M-CHAT

\begin{tabular}{ccccc}
\hline Ítems & $\mathbf{N}$ & $\mathbf{R}$ & $\mathbf{p}$ & Nivel \\
\hline 1 & 78 & 0,075 & 0,513 & Nula \\
2 & 78 & 0,186 & 0,102 & Nula \\
3 & 78 & $0,495^{* *}$ & 0,000 & Moderado \\
4 & 78 & 0,159 & 0,164 & Nula \\
5 & 78 & $0,465^{* *}$ & 0,000 & Moderado \\
6 & 78 & $0,697 * *$ & 0,000 & Moderado \\
7 & 78 & $0,644^{* *}$ & 0,000 & Moderado \\
8 & 78 & $0,317^{* *}$ & 0,005 & Débil \\
9 & 78 & $0,538^{* *}$ & 0,000 & Moderado \\
10 & 78 & $0,638^{* *}$ & 0,000 & Moderado \\
11 & 78 & $0,277^{*}$ & 0,014 & Débil \\
12 & 78 & $0,310^{* *}$ & 0,006 & Débil \\
13 & 78 & 0,063 & 0,586 & Nula \\
14 & 78 & $\mathrm{~B}$ &. & \\
15 & 78 & 0,001 & 0,992 & Nula \\
16 & 78 & $0,435^{* *}$ & 0,000 & Moderado \\
17 & 78 & $0,307^{* *}$ & 0,006 & Débil \\
18 & 78 & $0,237^{*}$ & 0,037 & Débil \\
19 & 78 & $0,307 * *$ & 0,006 & Débil \\
20 & 78 & $-0,124$ & 0,278 & Nula \\
21 & 78 & 0,124 & 0,278 & Nula \\
22 & 78 & $\mathrm{~B}$ &. & \\
23 & 78 & $0,616^{* *}$ & 0,000 & Moderada \\
\hline Nota: **correlación & significativa & en el nivel & 00.1 *correlación \\
significativa en el nivel 0.05 b. no se puede calcular, una de las \\
variables es constante. & & & \\
& & & &
\end{tabular}

interna, con 21 ítems pues para este análisis se eliminaron los dos ítems (14 y 22), que tuvieron una varianza cero. El valor de KR20 fue igual a 0,626, lo que indica un nivel de consistencia interna media para la puntuación total. En la dimensión de alteraciones en el desarrollo, el KR20 fue 0,396; en la dimensión de retraso o anormalidades en el lenguaje y la comunicación el KR20 fue 0,573 y en la dimensión de intereses restringidos y repetitivos el KR20 fue $-0,104$. La correlación test-retest con un intervalo de tres meses fue de $0,474(p<0,001)$.

\section{DISCUSIÓN}

En cuanto a la validez de contenido, utilizando el criterio de expertos, se determinaron valores positivos del M-CHAT, lo que señala una garantía psicométrica tal como refiere Escurra (1998). Sin embargo, dos ítems mostraron coeficientes $\mathrm{V}$ por debajo de 0,80, siendo no significativos. En relación a las dimensiones del instrumento, se ha obtenido que el total de los ítems en la dimensión alteraciones en el desarrollo de la interacción social, la respuesta emocional y el juego, han alcanzado un nivel alto y estadísticamente significativo. En la dimensión de retraso o anormalidades en el desarrollo del lenguaje y comunicación se encontró un total de siete ítems con los criterios necesarios pero dos ítems (17 y 21) no alcanzaron el nivel de significación adecuado. Finalmente, en la dimensión de intereses restringidos, movimientos repetitivos y alteraciones motrices se encontró que el total de los ítems han alcanzado un nivel alto y estadísticamente significativo en el coeficiente V. En resumen, estos resultados indican que el M-CHAT presenta en general un buen nivel de contenido, de acuerdo con el juicio de profesionales expertos en la evaluación del autismo.

Respecto de la validez clínica, se encontraron un índice de sensibilidad de 0,87 y de especificidad de 0,83 , ambos ubicados en un nivel alto. Estos altos valores indican que el instrumento es bastante preciso, al identificar correctamente la mayoría de los casos positivos y descartar correctamente la mayoría de los casos negativos. Los valores predictivos se encuentran entre un nivel aceptable y muy bueno. El valor predictivo positivo, que representa la probabilidad de que alguien con un resultado positivo en la prueba tenga la característica de interés fue de 0,64 ; mientras que el valor predictivo negativo, el cual representa la probabilidad de que alguien con un resultado negativo en la prueba no tenga la característica de interés fue de 0,95 . Ambos niveles nos otorgan valores positivos y significativos para determinar la validez clínica.

Por otro lado, utilizando el método de análisis de ítems, se encontraron que 14 de los 23 ítems correlacionan de manera significativa con el puntaje total. Por otro lado, se encontraron dos ítems para las que se pudo hacer este cálculo por presentar valores constantes en ellas, ítem 14 e ítem 22. Los ítems que no correlacionaron con la puntuación total probablemente reflejan características no relevantes al diagnóstico de autismo en la población de estudio.

Las correlaciones con el ADOS-2 y con el sistema portátil costo efectivo, si bien fueron positivas no resultaron significativas. Estos resultados apuntan, a primera vista, a que las pruebas utilizadas en 
este proceso no se encuentran evaluando el mismo constructo; en el caso del M-CHAT determina características específicas del desarrollo que se encuentran afectadas en TEA, en cambio el sistema portátil costo-efectivo sólo se encuentra determinando la preferencia visual para imágenes sociales o imágenes abstractas en movimiento, aun cuando se supone que este indicador es altamente significativo para el diagnóstico de autismo. Es probable, sin embargo, que este indicador por sí sólo no sea suficiente para predecir otros aspectos del autismo medidos por el M-CHAT. Por otra parte, la falta de correlación entre el M-CHAT y el ADOS-2 probablemente sea, también, el resultado de las diferencias entre los instrumentos tipo cuestionario que reportan conductas y las pruebas de desempeño realizadas por el propio sujeto. Sin embargo, tampoco puede descartarse en esta falta de correlación el hecho de que varios ítems del M-CHAT no correlacionan con la puntuación total.

Para la consistencia interna, se ha obtenido un valor de KR20 igual a 0,626, señalando un nivel de consistencia interna media o regular, encontrándose además KR20 de nivel débiles de 0,396 para la dimensión $\mathrm{A}$, moderado de 0,573 para la dimensión B y nulo de -0,104 para la dimensión C. De acuerdo con estos resultados, las dos primeras dimensiones se presentan como las más robustas y confiables y por tanto, posiblemente aptas para su utilización. En el caso de la tercera dimensión, un factor que podría haber contribuido a su baja confiabilidad es el número pequeño de ítems (3). La correlación test-retest hallada indica un nivel moderado de estabilidad en un plazo relativamente largo (tres meses).

Un aspecto a destacar del presente estudio radica en las edades consideradas en la muestra investigada. En la toma de esta decisión se tomó en cuenta los rangos de edad en que los niños son diagnosticados en el Perú, aunque algunas características analizadas en los ítems no se encuentran presentes en estas edades. Esto último puede haber contribuido también a la baja confiabilidad observada en la dimensión $\mathrm{C}$ así como a las bajas correlaciones con los otros instrumentos de diagnóstico aquí utilizados.

\section{Correspondencia}

Daniela Hidalgo Medina

Correo electrónico: rosa.pizarro@upch.pe

\section{REFERENCIAS}

American Psychiatric Association. (2013). Diagnostic and statistical manual of mental disorders. Washington D.C: American Psychiatric Association. Canal, R. (2007). Detención y Diagnóstico de trastornos del espectro autista. Madrid: Real Patronato sobre Discapacidad.

Centers for Disease Control. (S.f.). Data \& Statistics on Autism Spectrum Disorder. Washington: Centers for Disease Control. Recuperado de: http://www.cdc. gov/ncbddd/autism/data.html

Consejo Nacional de la Igualdad de las Discapacidades. (2014). Manual para Padres de Niños Autistas. Lima: Consejo Nacional de la Igualdad de las Discapacidades. Recuperado de: http://www.conadisperu.gob.pe/

Díez-Cuervo, M. (2005). Guía práctica para el diagnóstico de los trastornos del espectro autista. Revista de Neurología, 5, 299-310.

Escurra, M. (1998). Cuantificación de la validez de contenido por criterio de jueces. Revista de Psicología, 6, 103-111

Filipek, P.A. (1999). The screening and diagnosis of autistic spectrum disorders. Journal of Autism and Developmental Disorders, 29(6), 439-484.

Lord, C., Rutter, M., DiLavore, P. C., Risi, S., Gotham, K. \& Bishop, S. L. (2015). ADOS-2. Escala de Observación para el Diagnóstico del Autismo - 2 . Manual (Parte I): Módulos 1-4 (T. Luque, adaptadora). Madrid: TEA Ediciones.

Manzone, L. A. (2013). Adaptación y validación del Modified Checklist for Autism in Toddlers para población urbana argentina. Psicodebate, 13, 79-105.

Ministerio de la Mujer y Poblaciones Vulnerables. (2014). Reglamento de Ley $N^{\circ} 30150$. Lima: Ministerio de la Mujer y Poblaciones Vulnerables. Recuperado de: http://www.mimp.gob.pe/files/transparencia/ resoluciones_ministeriales/Proyecto-de-Reglamento-dela-Ley-30150.pdf

Baio, J. (2010). Prevalence of Autism Spectrum Disorder Among Children Aged 8 Years- Autism and Developmental Disabilities Monitoring Network, 11 Sites, United States, 2010. Surveillance Summaries, 63(SS02), 1-21. Obtenido de http://www.cdc. gov/mmwr/preview/mmwrhtml/ss6302a1.htm?s cid $=$ ss6302a $1 \mathrm{~W}$

Posada-De la Paz, M. (2005). Investigación epidemiológica en el autismo: una visión integradora. Revista de Neurología, 40, 191-198.

Riviére, A. (1997). Tratamiento y definición del espectro autista I y II. En: J. A. Riviére. El tratamiento del autismo. Nuevas perspectivas (pp. 61-160). Madrid: Ministerio de Trabajo y Asuntos Sociales.

Rivière, A. (2000). ¿Cómo aparece el autismo? Diagnóstico temprano e indicadores precoces del trastorno autista. En: A. Rivière \& J. Martos J. (Eds). 
El niño pequeño con autismo. (pp. 13-32). Madrid: APNA-IMSERSO.

Robins, D., Fein, D., Barton, M. L. \& Green, J. A. (2001). The Modified Checklist for Autism in toddlers: An initial study investigating the early detection of autism and pervasive developmental disorders. Journal of Autism and Developmental Disorders, 31, 131-144.

Sampedro, M. (2012). Detección temprana de autismo ¿es posible y necesaria? CES Psicología, 5(1), 112-117.
Suárez, X. (1994). Perfil de stress de madres y padres cuyos hijos presentan el diagnóstico de autismo y son atendidos en el centro de educación especial Ann Sullivan. Lima. Tesis para optar el Título Profesional de Licenciada en Psicología. Departamento de Psicología, Universidad Peruana Cayetano Heredia.

Villalba, J. (2013). Validación del Q-Chat para detectar el trastorno autista en edades tempranas. Tesis de Maestría en Psicología. Bogotá: Universidad Nacional de Colombia. 